\title{
Functional intertwining of Dpp and EGFR signaling during D rosophila endoderm induction
}

\author{
Dávid Szüts, Salih Eresh, and Mariann Bienz ${ }^{1}$ \\ Medical Research Council (MRC) Laboratory of Molecular Biology, Cambridge CB2 2QH, UK
}

\begin{abstract}
Endoderm induction in D rosophila is mediated by the extracellular signals Decapentaplegic (Dpp) and Wingless (Wg). We discovered a secondary signal with a permissive role in this process, namely Vein, a neuregulin-like ligand that stimulates the epidermal growth factor receptor (EGFR) and Ras signaling. Dpp and Wg up-regulate vein expression in the midgut mesoderm in two regions overlapping the Dpp sources. Experiments based on lack of function and ectopic stimulation of Dpp and EGFR signaling show that these two pathways are functionally interdependent and that they synergize with each other, revealing functional intertwining. The transcriptional response elements for the Dpp signal in midgut enhancers from homeotic target genes are bipartite, comprising CRE sites as well as binding sites for the Dpp signal-transducing protein Mad. Of these sites, the CRE seems to function primarily in the response to Ras, the secondary signal of Dpp. We discuss the potential significance of why an inductive process might use a secondary signal whose function is intertwined with that of the primary signal.
\end{abstract}

[Key Words: Epidermal growth factor receptor; vein; decapentaplegic; M ad binding sites; endoderm induction]

Received December 30, 1997; revised version accepted A pril 21, 1998.

Drosophila Decapentaplegic (Dpp) and vertebrate activins are related extracellular signaling molecules with major organizational roles during animal development. For example, Dpp patterns the dorsoventral axis in the early fly embryo (Irish and Gelbart 1987) as well as the adult appendages (Spencer et al. 1982; Zecca et al. 1995). Similarly, activin-like signals are thought to function during axis formation in the early frog embryo, in particular, during induction and patterning of the mesoderm (Smith et al. 1990; Hemmati-Brivanlou and M el ton 1992; Kessler and Melton 1995). Perhaps the most remarkable property of these signal s is their capacity to act across I arge cellular distances and at multipl e threshol ds to elicit distinct cellular responses (Green and Smith 1990; Ferguson and Anderson 1992; Green et al. 1992; Gurdon et al. 1994; Lecuit et al. 1996; N ellen et al. 1996). They have thus been referred to as morphogens (Lawrence and Struhl 1996).

Dpp al so has a prime function during endoderm induction in Drosophila (Bienz 1997; Fig. 1). Dpp is secreted from the outer cell layer of the embryonic midgut, the visceral mesoderm, where its main source of expression in parasegment ps7 depends directly on the homeotic gene Ultrabithorax (U bx) (Sun et al. 1995). In the same cell layer, Dpp stimulates expression of another extracellular signal, Wingless (Wg), in a neighboring paraseg-

${ }^{1}$ Corresponding author.

E-MAIL mb2@mrc-Imb.cam.ac.uk; FAX UK 1223412142. ment (ps8; Immerglück et al. 1990) that in turn feeds back to ps7 to stimulate $U$ bx expression. Thus, Dpp is part of a "parautocrine" feedback loop of U bx (i.e., an autocrine feedback loop based partly on paracrine action; see Bienz 1997) that sustains its own expression through Dpp and Wg (Hursh et al. 1993; Thüringer and Bienz 1993). D pp also spreads to the inner layer of the embryonic midgut, the endoderm, where it synergizes with $\mathrm{Wg}$ to induce expression of the homeotic gene labial (lab) (Immerglück et al. 1990; Panganiban et al. 1990; Reuter et al. 1990). To achieve this, Dpp locally el evates the endodermal expression levels of Drosophila D-Fos with which it cooperates to induce lab (Riese et al. 1997a). Differentiation of various cell types in the larval gut depends on these inductive effects of D pp and Wg (Hoppler and Bienz 1994, 1995).

We reported recently that a CAMP response element (CRE) from the $U$ bx midgut enhancer is necessary and to some extent sufficient to mediate the Dpp response in the embryonic midgut (Eresh et al. 1997). CREs are known to be signal-responsive elements, not only for CAM P signaling as described initially (Montminy et al. 1986) but also for other signals including ones acting through Ras (e.g., de Groot et al. 1993; Ginty et al. 1994). This prompted us to ask whether any other signal may play a part in the Dpp response. This led us to discover that the Drosophila epidermal growth factor receptor (EGFR) has a critical function during endoderm induction. 
\begin{tabular}{|l|l|l|l|l|l|l|l|l|l|l|l}
2 & 3 & 4 & 5 & 6 & 7 & 8 & 9 & 10 & 11 & 12 & ps
\end{tabular}

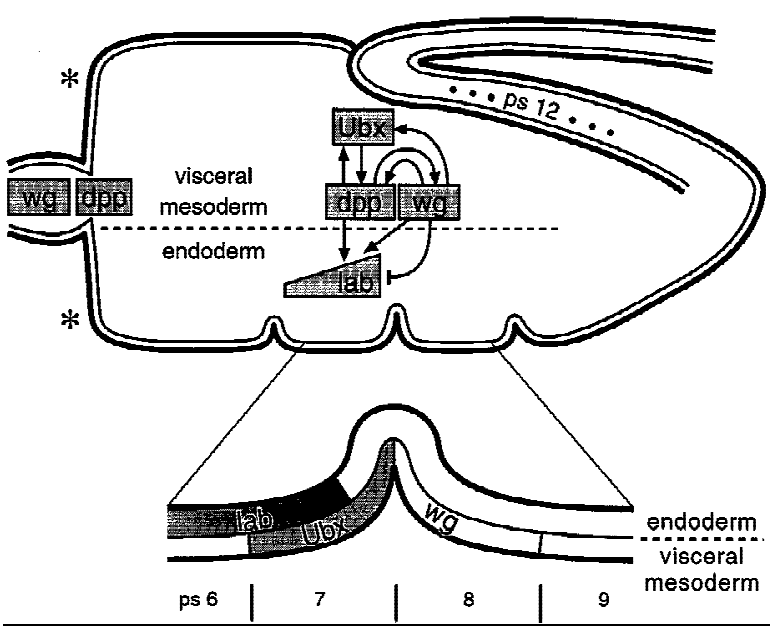

Figure 1. Endoderm induction. The embryonic midgut is drawn schematically in relation to parasegmental position (ps); outer cell layer, visceral mesoderm; inner cell layer, endoderm. The three constrictions are positioned at the junctions between ps5/ps6, ps7/ps8, and ps9/ps10; the proventriculus spans approximately ps2, and the gastric caeca are budding in ps3 [asterisks $\left(^{*}\right)$ ]; the midgut ends posteriorly to ps12 (ps12 is stretched at this stage as indicated by dots). Within the midgut outline, the main genes mediating endoderm induction and their regulatory relationships are sketched out (note that D-Fos was omitted, for clarity; see Riese et al. 1997a). Underneath the drawing, the critical region of ps6-ps9 is blown up to highlight the spatial relationships of $\mathrm{Ubx}, \mathrm{Lab}$, and $\mathrm{Wg}$ expression (for references, see text).

Here, we provide evidence that EGFR is stimulated by its ligand Vein whose expression is up-regulated locally in the visceral mesoderm, in regions overlapping the D pp sources. We show that this up-regulation depends on dpp and wg. Vein is thus a secondary signal of Dpp and Wg, and we show that it stimulates homeotic gene expression in both cell layers of the midgut. Finally, our results suggest an intimate functional connection between Dpp and EGFR signaling in that they are functionally interdependent and that they synergize with each other.

\section{Results}

EGFR signaling is required for lab induction in the embryonic midgut

Loss-of-function mutants of the Drosophila EGFR are very abnormal and do not develop properly beyond the early embryonic stages (Clifford and Schüpbach 1992; Raz and Shilo 1992; D. Szüts, S. Eresh, and M. Bienz, unpubl.). We therefore used a temperature-sensitive allele of EGFR, $\mathrm{fl}^{1 \mathrm{~F} 26}$, to ask whether this receptor has any function in the embryonic midgut. We stained $\mathrm{flb}^{1 \mathrm{~F} 26}$ embryos with Lab antibody after shifting them from the permissive to the restrictive temperature at 6-8 hr of development (i.e., before midgut formation, but allowing normal germ-band retraction). We found that the midguts of the homozygous $\mathrm{flb}^{1 \mathrm{~F} 26}$ embryos were severely abnormal, with none of the constrictions forming properly, and that they showed virtually no Lab staining in the midgut epithelium (Fig. 2B). These phenotypes indicate a critical function of EGFR in the embryonic midgut.

We noted that many endodermal cells were missing or seemingly unhealthy, especially in the middle midgut where lab is induced and in the anterior midgut near the gastric caeca. N ote that these two midgut regions correspond to the domains of Dpp expression (Fig. 1). Similar effects of EGFR loss of function on cell heal th have been observed in earlier studies of the embryonic epidermis (Clifford and Schüpbach 1992; Raz and Shilo 1992). Although this putative function of EGFR in cell survival may contribute to the observed loss of lab induction, we believe that it does not account for all aspects of the gut phenotypes attributable to EGFR Ioss of function (see below).

We further studied the function of EGFR by examining the effects of GAL4-mediated overexpression of a dominant-negati ve version of EGFR [called DN -DER (O'Keefe et al. 1997); DN-DER is a truncated EGFR that lacks the intracellular kinase effector domain]. DN-DER has been shown to interfere with endogenous EGFR function in the eye-imaginal disc (Freeman 1996) and in the em-

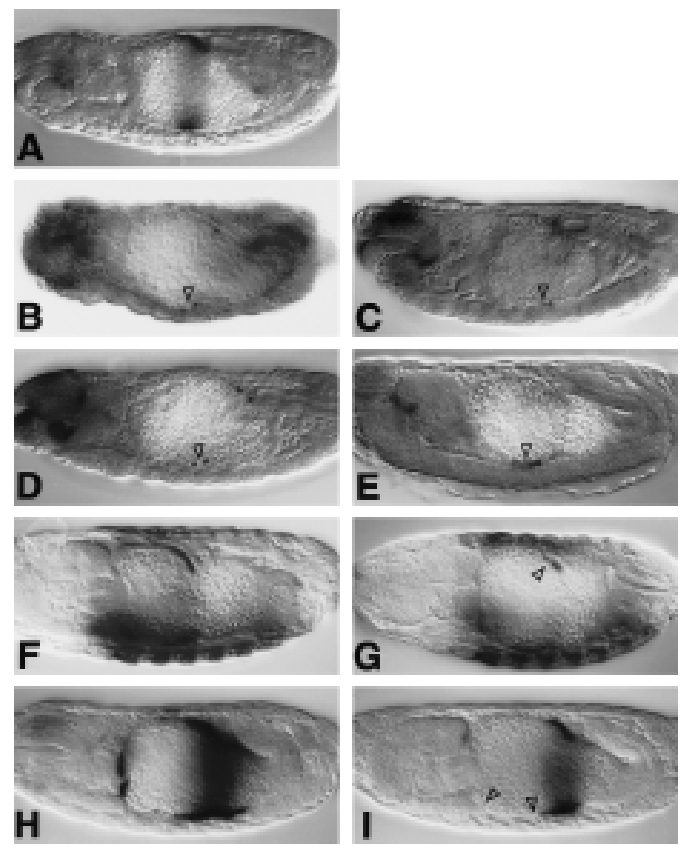

Figure 2. Requirements of EGFR and vein during endoderm induction. Side views of 12- to 14-hr-old embryos stained with Lab (A-E) or Ubx antibody ( $F, G)$ or with lacZ antibody to visualize $U$ bx $B$ expression $(H, I)$. Wild type $(A, F, H)$; flb ${ }^{1 F 26}$ homozygote (B); 48Y.GAL4/UAS.DN -DER (C); 24B.GAL4/UAS.DN DER (G,I); vein ${ }^{\gamma 4}$ homozygote (D); vein ${ }^{\gamma 3} / \operatorname{vein}^{\mathrm{P} 1749}(\mathrm{E})$. N ote the loss of Lab staining and the reduced $U$ bx staining ( $\triangle$ in $B-E, G$ ) and the loss of $U$ bx B-mediated expression in ps6/ps7 and in ps3 ( $\triangle$ in I), owing to lack of function of EGFR or vein. Anterior to the left, dorsal up (orientation the same in all figures). 
bryonic epidermis (O'Keefe et al. 1997; Szüts et al. 1997). This allowed us to block EGFR function selectively in the visceral mesoderm (after overexpression with 24B.GAL4; Brand and Perrimon 1993) or in the endoderm (after overexpression with 48Y.GAL4; MartinBermudo et al. 1997), thus avoiding many of the drastic consequences of I osing EGFR function in the whole embryo (with $\mathrm{fl}^{1 \mathrm{~F} 26}$ ). We found that, after ubiquitous expression of DN-DER in the endoderm, Lab expression in the midgut was severely reduced (Fig. 2C). We can only detect Lab staining in a few cells in the middle midgut, typically scattered throughout the endodermal domain in which Lab expression is normally seen (the lab domain; Fig. 2A). As in the flb ${ }^{1 F 26}$ embryos, cells in the endoderm seem to be missing or unhealthy in the lab domain and also in the anterior midgut in the ps3/ps4 region; however, the midgut epithelium still seems to be largely intact, judging by the expression pattern of an endodermal marker gene (see Materials and M ethods). This result indicates a function of EGFR in the endoderm.

We al so observed a reduction of Lab expression, al beit less severe, after mesodermal expression of DN-DER (not shown). This suggested that EGFR functions in the visceral mesoderm too. To examine this further, we stained embryos in which DN-DER was produced in the mesoderm with antibodies against $\mathrm{Ubx}$ and $\mathrm{Wg}$. Ubx staining in the visceral mesoderm is somewhat reduced in these embryos (Fig. 2G, cf. to F), as is staining for Wg in this cell layer (not shown). We also found that DNDER affected the midgut morphology under these conditions: The constrictions were abnormal, and the gastric caeca tended to be stunted.

Clearly, the effects of DN-DER on gene expression in the visceral mesoderm are slight compared with its effects on Lab expression in the endoderm. This parallels the effects of dpp and wg mutations that only slightly reduce gene expression in the visceral mesoderm while strongly diminishing Lab expression (Immerglück et al. 1990; Panganiban et al. 1990). The stimulatory effects of dpp and wg on $U$ bx expressi on were revealed much more clearly by $\beta$-galactosidase (lacZ) reporter genes contai $n$ ing dpp- and wg-responsi ve enhancer elements (Hursh et al. 1993; Thüringer and Bienz 1993). We thus examined the activity of the minimal $U$ bx midgut enhancer ( $U$ bx B) after mesodermal expression of DN-DER.

U bx B normally mediates strong lacZ staining in a regi on spanning the middle midgut constriction, in ps6ps9, and al so some staining in the gastric caeca, in ps3 (Fig. 2H; Thüringer et al. 1993); the strongest staining in ps7/ps8 spans the main Dpp and Wg sources in the middle midgut, whereas the ps3 staining coincides with the anterior source of Dpp (Figs. 1 and 5B, below). Mesodermal expression of DN-DER almost completely eliminates staining in ps3 and strongly reduces staining in the ps6/ps7 region (open triangles in Figs. 21 and 4F, below). These results lend strong support to our notion that EGFR functions in the visceral mesoderm; they indicate that EGFR positively regulates $U$ bx expression.

Finally, we asked which ligand might activate EGFR in the two cell layers of the midgut. Two ligands are known that activate EGFR in somatic cells of Drosophila: Spitz, which apparently needs to be processed to an active form by the membrane-spanning protein Rhomboid (Bier et al. 1990; Rutledge et al. 1992; Freeman 1994; Schweitzer et al . 1995a; Golembo et al. 1996a; Gabay et al. 1997), and Vein (Schnepp et al. 1996; Simcox et al. 1996; Simcox 1997; Yarnitzky et al. 1997). A third EGFR ligand, Gurken, is known, but its function is restricted to the germ line (N euman-Si l berberg and Schüpbach 1993). We examined spitz and rhomboid loss-offunction mutants (see Materials and M ethods) by staining embryos with Lab antibody, but these mutants showed only a minor effect on Lab expression: Typically, we found Lab staining to be missing in just a few cells in the lab domain, and the midgut constrictions are normal in these mutants.

However, vein mutant embryos show a drastic effect on Lab expression. The most extreme mutant conditions (see $M$ aterials and $M$ ethods) caused nearly complete loss of Lab staining in the midgut; none of the midgut constrictions formed (Fig. 2D), nor did the gastric caeca el ongate (not shown). Milder mutant conditions have only sporadic effects in the midgut as only some cells in the lab domain lack Lab expression; the constrictions and the gastric caeca form normally under these conditions (Fig. 2E). These results implicate Vein as a critical ligand of EGFR in the embryonic midgut.

Vein expression is up-regulated in the Dpp domains by dpp and wg

EGFR expression is thought to be fai rly ubiquitous in the embryo (Zak et al. 1990). However, vein transcripts are found in a highly restricted pattern, primarily in the embryonic mesoderm (Schnepp et al. 1996; Yarnitzky et al. 1997; D. Szüts, S. Eresh, and M. Bienz, unpubl.). We found that, in the midgut too, vein expression is spatially regulated, as follows:

vein transcripts in the midgut are restricted to the visceral mesoderm. Initially, during stage 13 (for stages, see Campos-Ortega and Hartenstein 1985), low levels of vein expression are seen at intervals throughout the midgut mesoderm. However, soon after the formation of the midgut epithelium, vein transcripts start to accumulate locally, and two main domains of prominent vein expression develop, one in the anterior and one in the middle midgut (Fig. 3A). Anteriorly, vein expression spans approximately ps2-ps4 and is strongest around the ps3/ps4 junction, that is, posteriorly to the gastric caeca. In the middle midgut, there is a fairly wide band of low vein expression spanning approximately ps6-ps10, with strongly up-regulated expression levels throughout ps7 (and trailing into anterior ps8). Posterior ps7 becomes the most prominent site of vein expression in the midgut. Finally, a narrow band with low levels of vein transcripts is seen at the posterior end of the midgut. We note that the two main expression domains of vein overlap the two domains of Dpp expression in the visceral mesoderm (in ps3 and ps7; Fig. 1), but each of them is considerably wider than the corresponding dpp domain. 


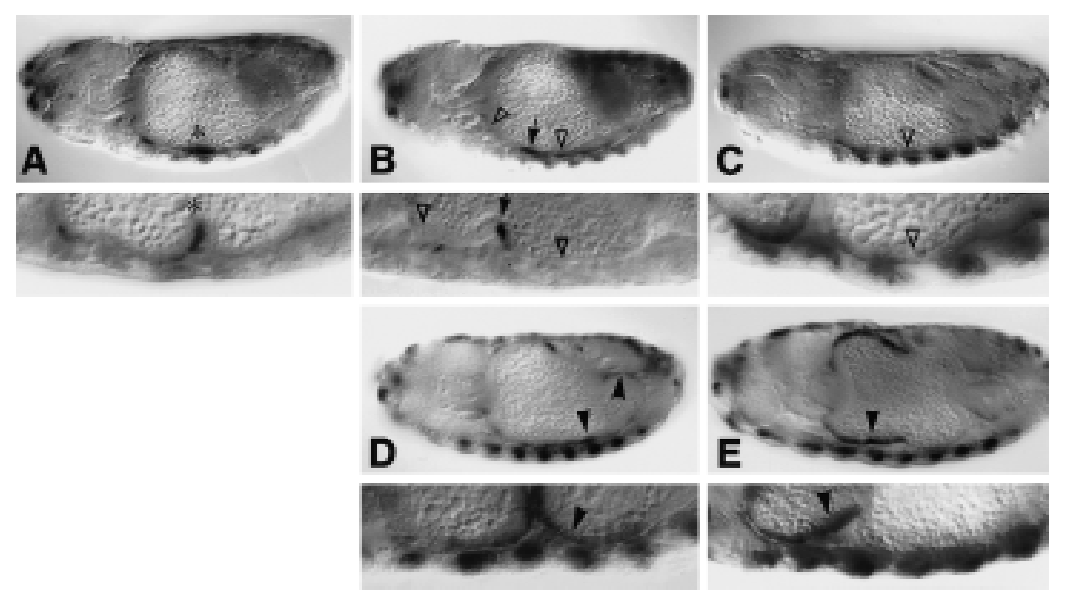

Figure 3. Expression of vein transcripts in wildtype and mutant midguts. Side views of $12-$ to 15-hr-old embryos, after in situ hybridization to vein transcripts: Wild type (A), dpp ${ }^{\mathrm{s4}}$ homozygote (B), wg ${ }^{\prime L 114}$ homozygote (C), 24B.GAL4/UAS.Dpp (D), 24B.GAL4/UAS.Wg (E); underneath each picture, a high-magnification view is shown of the corresponding genotype at a slightly older stage, to highlight the ventral ps3-ps10 region of the midgut. Maximal vein expression in the wild type is seen in posterior ps7, just anterior to the middle midgut constriction [indicated by asterisks $(*)$ in A]. N ote the reduction of vein expression in the ps7 and ps4 regions in dpp and wg mutants ( $\triangle$ in $B$; in $C$, only the reduction in ps7 is indicated by $\triangle$ as the reductive effect in ps4 of wg mutants is mild) and also the novel expression in the first constriction of dpp mutants (arrows in B). Additional vein expression is visible in the ps8-ps10 and ps12 regions after Dpp expression (arrowheads in D) and in ps2-ps7 after Wg expression throughout the midgut (arrowheads in E).

Dpp expression is barely reduced in vein mutant embryos (not shown). However, when we examined dpp ${ }^{\mathrm{s}}$ mutants, we found that vein expression in the visceral mesoderm is severely diminished (Fig. 3B). We no longer see the prominent band of vein expression in ps7, and expression in ps4 is reduced too. Instead, the strongest expression of vein in these mutants is seen at a novel location, at the ps5/ps6 junction around the incipient first midgut constriction (this ps5/ps6 expression is higher than in the wild type, and can be used to identify young dpp mutant embryos). We conclude that dpp is required for the localized up-regulation of vein expression in the midgut.

We asked whether vein expression is also under the control of wg. Using a temperature-sensitive allele of wg (allowing us to remove wg function during the critical phases of endoderm induction; Yu et al. 1996), we found that vein expression is also strongly diminished in $\mathrm{wg}$ mutants (Fig. 3C): We can still see vein expression at moderate levels in the ps4 region, but vein expression is barely visible elsewhere in the midgut of these mutants. In particular, there are only traces of vein expression in the ps7/ps8 region, and expression at both midgut ends is almost undetectable. Clearly, wg plays an essential role too in up-regulating vein expression.

We asked whether dpp and wg might be sufficient to position the two domains of vein up-regulation. We first examined the patterns of vein transcripts in embryos in which Dpp was expressed throughout the mesoderm. We found that vein expression was stronger in many regions of the midgut, notably in ps8-ps10 and throughout ps12 (Fig. 3D; the former is probably mediated in parts by endogenous $\mathrm{Wg}$, see below: $\mathrm{Wg}$ expression is seen throughout ps8-ps10 under these conditions; StaehlingHampton and Hoffmann 1994). Evidently, ectopic Dpp produces stronger and al so some ectopic vein expression, indicating a critical role of $\mathrm{dpp}$ in positioning vein upregulation.
We also examined vein expression after expressing $\mathrm{Wg}$ throughout the mesoderm. This condition of high $\mathrm{Wg}$ pathway activity throughout the midgut results in ectopic activation of endogenous dpp in ps2-ps7 (Yu et al. 1996), and stimulatory effects in this region are likely to be the result of combined $\mathrm{Wg}$ and Dpp signaling. High mesodermal $\mathrm{Wg}$ causes very strong vein expression in ps2-ps7 (Fig. 3E), significantly stronger than that caused in this region by mesodermal Dpp expression alone (Fig. 3D). This indicates that wg cooperates with dpp in positioning vein up-regulation.

We note that the response of vein to ectopic Dpp is very similar to that of $\mathrm{Ubx} \mathrm{B}$ (Figs. $4 \mathrm{G}$ and 5; see also Thüringer et al. 1993): U bx B responds to ectopic Dpp chiefly in three regions of the midgut (Figs. 4G and 5); in two of these, ectopic Dpp al so stimulates vein (ps8-ps10 and ps12; Fig. 3D), whereas in the third, vein expression is al ready high under normal conditions (ps2/ps3; Fig. 3A). Likewise, the response of $U \mathrm{bx} B$ to high ubiquitous $\mathrm{Wg}$ is indistinguishable from that of vein to high $\mathrm{Wg}$ (including repressive effects posteriorly to ps7; Fig. 3E; X. Yu, S. Eresh, J. Riese, and M ariann Bienz, in prep.). This suggests that $U$ bx $B$ responds to ectopic Dpp or Wg only in cells that themselves express high levels of vein or that are near vein-expressing cells.

In summary, these results are strong evidence that $\mathrm{dpp}$ and wg position the two main domains of strong vein expression in the midgut mesoderm and that they stimulate vein expression in these domains. This places vein downstream of $\mathrm{dpp}$ and $\mathrm{wg}$ in the inductive cascade (Fig. 8, below). Furthermore, the strong effects of vein and EGFR loss of function on Lab expression place Vein and EGFR upstream of lab. Finally, the effects of vein and EGFR loss of function on the $U$ bx enhancer and on $\mathrm{U}$ bx expression itself are consistent with a function of Vein and EGFR in the parautocrine feedback loop of Ubx. 


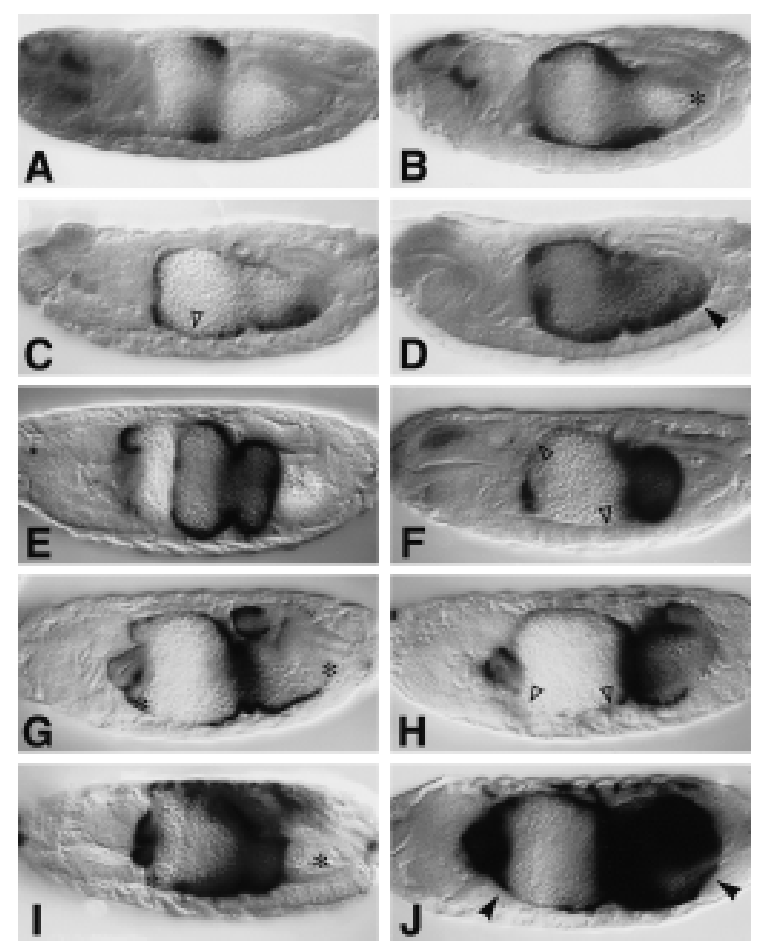

Figure 4. Interdependence and synergy between EGFR and Dpp signaling. Side views of 12- to 15-hr-old embryos stained with Lab antibody (A-D) or with LacZ antibody to visualize U bx B expression (E-J): Wild type (A,E); 48Y.GAL4/UAS.Dpp (B); 48Y.GAL4/UAS.Dpp/UAS.DN -DER (C); 48Y.GAL4/UAS.Dpp/UAS.Dras1 ${ }^{\mathrm{V} 12}$ (D); wild type (E); 24B.GAL4/UAS.DN DER (F); 24B.GAL4/UAS.Dpp (G); 24B.GAL4/UAS.Dpp/ UAS.DN-DER (H); 24B.GAL4/UAS.Dras1 ${ }^{\mathrm{V} 12}$ (I); 24B.GAL4/ UAS.Dpp/UAS.Dras1 ${ }^{\mathrm{V} 12}$ (J). DN -DER antagonizes the stimulatory effects of $D p p$ in the anterior midgut ( $\triangle$ in $C, F, H$; cf. with $B, E, G$, respectively), whereas Dras ${ }^{\mathrm{V} 12}$ synergizes with $D p p$ [as judged by conspicuously strong and novel expression marked by arrowheads in D, J; cf. with the regions marked by asterisks $\left(^{*}\right)$ in $B, G, I$ in which only low or no expression is seen in embryos expressing Dpp or Dras1 ${ }^{\mathrm{V} 12}$ alone].

\section{Functional intertwining of EGFR and Dpp signaling}

Recall that the main effects of impaired EGFR or vein functions in the midgut are seen in ps7 and ps3, near the Dpp sources. Recall al so that the $U$ bx B enhancer seems to respond to Dpp only in or near vein-expressing cells. These observations suggest that EGFR/vein is required for Dpp to be effective in stimulating midgut gene expression.

We tested this further by monitoring $\mathrm{U}$ bx $\mathrm{B}$ expression after coexpressing Dpp with DN-DER throughout the mesoderm or by monitoring Lab expression after coexpressing Dpp with DN-DER in the endoderm. In both cases, we found that DN-DER substantially reduced the activity of ectopic Dpp: Although ectopic mesodermal D pp produced ectopi $\mathrm{c}$ lac Z staining from U bx B in various regions of the midgut mesoderm (Fig. 4, cf. G and E), staining is much reduced in the ps6/ps7 region, in ps3/ ps4, and in ps12, after coexpression with DN-DER (Fig. 4 , cf. $\mathrm{H}$ and $\mathrm{G}$ ). Likewise, whereas ectopic endodermal
Dpp strongly induces Lab staining through much of the endoderm (Fig. 4B), this staining is clearly reduced in most endodermal cells after coexpression with DN -DER (Fig. 4C, open triangle marks the ps4-ps6 region that is most affected; there is al so some widening of Lab staining posteriorly to approximately ps9 in these embryos, to be discussed el sewhere). This suppressive effect of DN DER is not detectable until later stages of endoderm induction, probably because the levels of DN -DER need to build up to interfere effectively with endogenous EGFR. We note that the gut morphology of the DN-DER/Dpp embryos is more normal than that of the Dpp embryos, suggesting that DN-DER also suppresses some of the phenotypic effects of ectopic D pp on constriction formation. These results show that DN-DER suppresses Dppinduced gene expression and phenotypic effects in many regions of the midgut. This suppression is particularly significant in gut regions in which DN-DER overexpression does not detectably affect cell heal th and shape (i.e., outside the realms of endogenous Dpp, see above). The results strongly support our notion that $D p p$ signaling is ineffectual in the absence of EGFR signaling.

Then, we asked the converse, namely whether EGFR signaling could function in the absence of D pp signaling. To stimulate EGFR throughout the midgut, we first overexpressed Vein in the mesoderm or endoderm, but we did not see any effects on $U$ bx $B$ or on Lab expression. However, the effects of ectopic Vein were found to be very weak in our most sensitive assay system, in the wing imaginal disc (not shown). We therefore resorted to overexpressing a constitutive form of Drosophila Ras1 (Dras1 $^{\mathrm{V} 12}$; Lee et al. 1996) that we previously found to mimic strong constitutive EGFR signal ing in the embryonic epi dermis (Szüts et al . 1997). We did not detect any significant effects of endodermally expressed Dras $1^{\mathrm{v} 12}$ on Lab expression (not shown). However, when we monitored lacZ staining from $U$ bx B after mesodermal Dras $1^{\mathrm{V} 12}$ expression, we found that staining was substantially enhanced (Figs. 4, cf. I and E, and 6 cf. C and B). This increase in staining intensity is confined to cells in which $U$ bx $B$ is normally active; it is most prominent in regions in which lacZ staining in the wild type is weak, for example, in ps6 (Fig. 6C, arrowheads) and in the dorsal somatic mesoderm (Fig. 6C, arrows). There are no major effects on the gut morphology of Drasi ${ }^{\mathrm{V} 12} \mathrm{em}$ bryos, but we noticed that the formation of the first constriction is often delayed or even suppressed. Thus, Dras $1^{\mathrm{V} 12}$ has a significant stimulatory effect on $U$ bx $B$ expression, but Dras $1^{\mathrm{V} 12}$ is incapable of inducing $U$ bx $B$ or Lab expression ectopically. This indicates a permissive role of EGFR signaling in the midgut.

These results suggest that neither Dpp nor EGFR signaling is particularly effective in the absence of the other, but each pathway seems to be capable to elicit some response on its own (see also below). We thus asked whether they would synergize if coactivated ectopically. We coexpressed Dpp and Dras1 $1^{\mathrm{V} 12}$ throughout the mesoderm and examined the effects of this condition on $\mathrm{U}$ bx B. IacZ staining in the midgut of these embryos is very strong, much stronger than would be expected 
from addition of expression attributable to $\mathrm{Dpp}$ or Dras1 $^{\mathrm{V} 12}$ al one (Fig. 4, cf. J. and G or I). For example, we see strong lacZ staining between ps9 and ps12 or in the ps3/ps4 region (arrowheads in Fig. 4J), where there is virtually no (or only little) expression if either of them is overexpressed al one (asterisks in Fig. 4G,I). Encouraged by this, we also stained embryos coexpressing Dpp and $\operatorname{Dras}^{\mathrm{V} 12}$ with Lab antibody. We found that there is strong Lab staining posteriorly to ps8/ps9 under these costimulation conditions (arrowhead in Fig. 4D), whereas there is virtually no staining in this midgut region in embryos expressing Dpp or Dras1 ${ }^{\mathrm{V} 12}$ alone (asterisk in Fig. 4B). Thus, there are strong synergistic effects between Dpp and Ras signaling in the visceral mesoderm and in the endoderm. In contrast, we did not detect any synergism between Ras and Wg signaling on U bx B (assayed in the same way; not shown).

In summary, we have made two observations that complement each other, namely, a functional interdependence of Dpp and EGFR signaling but also a strong synergism between these two pathways. This illustrates an intimate functional connection between the two signaling pathways in the embryonic midgut.

\section{A bipartite response el ement for Dpp and EGFR signaling in the $U$ bx enhancer}

We reported previously that the Dpp response sequence in U bx B maps to a CRE sequence: If this CRE is mutated, the mutant enhancer no longer responds to Dpp signaling; conversely, a minimal oligomer construct with multimerized CREs produces Dpp-responsive expression in the midgut (Eresh et al. 1997). Recently, Laughon and colleagues discovered that this CRE is adjacent to a binding site for the protein $\mathrm{Mad}$ (Kim et al. 1997). $M$ ad is encoded by mothers against dpp (mad), a gene known to be required for $D$ pp signal transduction in many, if not all, developmental contexts, including the embryonic midgut (Sekelsky et al. 1995; N ewfeld et al. 1996). M ad is the founding member of the Smad family of proteins that are found in vertebrates and invertebrates; most Smads are activated by TGF- $\beta$-like signals and, consequently, translocate into the nucleus (for review, see M assagué et al. 1997). The recent discovery of the DNA-binding properties of Drosophila M ad strongly suggested that $M$ ad is a transcription factor that mediates the stimulatory effects of D pp signaling directly by binding to enhancers of Dpp target genes (Kim et al. 1997).

Kim et al . (1997) also reported a minor Mad binding site (Mad A) within U bx B that overlaps the CRE and whose in vitro binding affinity to $M$ ad is 50-fold reduced compared with that of the main site ( $M$ ad $B$ ) adjacent to the CRE (Fig. 5A). However, this affinity was an underestimate as a result of a sequence error in $M$ ad $A$; using the correct sequence, we find that $M$ ad binds to $M$ ad $A$ and $M$ ad $B$ with comparable affinity (see $M$ aterials and M ethods). In the correct sequence, the best match to the $\mathrm{M}$ ad binding site consensus sequence (6 out of 7 resi- dues) overlaps the CRE almost entirely (Fig. 5A). Thus, our previous mutation in the Ubx CRE (called BC) is predicted to affect the CRE as well as Mad A, and both our minimal CRE oligomer constructs contain CREs as well as $M$ ad binding sites (5CRE contains $M$ ad $A$ and Mad B; 4CRE contains M ad A only; Eresh et al . 1997). We therefore wondered whether the $C R E$ has any function in the Dpp response or whether the $M$ ad binding sites are the true Dpp target sequences.

We tested this by introducing selective base substitutions into the $C R E$ and the $M$ ad binding sites. Given the overlap between $M$ ad $A$ and the $C R E$, it was not possible to mutate one without touching the other. We thus designed mutations that are predicted to disable one motif while leaving the other one essential ly intact: We introduced base substitutions into $M$ ad $B$ and $M$ ad $A$ with only a minimal alteration to the CRE (BM 2; Fig. 5A), and we mutated the CRE with only a minimal al teration to $M$ ad A (BC2; Fig. 5A). We al so generated BM 1 in which $M$ ad $B$ exclusively is mutated (Fig. 5A; BM 1 resembles our previous mutation B5; Eresh et al. 1997). All mutant motifs were tested in vitro for their binding to $M$ ad and also to dCREB-B (the only protein known to bind to the U bx CRE; Eresh et al. 1997). This confirmed that the $B M 1$ and BM 2 mutations abolish specific DNA binding of $\mathrm{M}$ ad, whereas $\mathrm{BC}$ and $\mathrm{BC} 2 \mathrm{did}$ not affect it; conversely, binding of dCREB-B was unaffected by BM 1 but was abolished by $B C$, strongly reduced by $B C 2$, and also reduced by BM 2 (see Materials and Methods). Bearing in mind the caveat that dCREB-B may not be the protein that acts through the U bx CRE in vivo (see Eresh et al. 1997), the dC REB-B binding data suggest that the CRE in $B C 2$ may retain residual activity and that the $C R E$ in BM 2 may not be fully active. Transgenic flies were made, and the activities of the mutant enhancers were tested in the wild type and in embryos with mesodermaIly expressed D pp or Dras $1^{\mathrm{V} 12}$, to assess their responsive ness to Dpp and EGFR signaling.

As expected from our previous mutant B5 construct (Eresh et al. 1997), BM 1 still mediates strong expression in the wild-type midgut, with some additional lacZ staining in ps10 (Fig. 6E). BM 1 is al so extensively Dpp responsive (Fig. 6D) as well as Ras responsive (Fig. 6F). Thus, mutation of $\mathrm{M}$ ad $\mathrm{B}$ al one causes neither substantial loss of expression nor of signal responsiveness. Mad $B$ is therefore not critical in responding either to Dpp or to Ras. On the contrary, the ectopic staining in ps10 indicates that BM 1 eliminates binding of a constitutive repressor to $\mathrm{M}$ ad $\mathrm{B}$. This repressor might be a protein different from $M$ ad recognizing a sequence overlapping $\mathrm{Mad} B$.

In contrast, BM 2 eliminates virtually all staining in ps6 and ps7 and in ps3 of the visceral mesoderm (Fig. $6 \mathrm{H})$, that is, in regions that coincide with Dpp sources. The remaining staining in ps8 and ps9 is also reduced compared with that produced by the wild-type enhancer in this region. Accordingly, BM 2 shows essentially no responsiveness to ectopic Dpp (Fig. 6G), except for some ectopic staining that is seen in ps10 and at both ends of the midgut, most probably reflecting the stimulatory ef- 
Figure 5. Targets for Vein/EGFR and Dpp signaling in the U bx B enhancer. (A) Sequences of wild-type (B) and mutant enhancers (BC, BC 2, BM 1, BM 2), underneath a sketch laying out the palindromic CRE and the two $M$ ad binding sites ( $M$ ad $A$ and $M$ ad $B$ ); matches to the $C R E$ or $M$ ad binding site consensus sequences (given at bottom) are indicated with horizontal bars in wild-type and mutant enhancers (for CRE above, for $M$ ad below sequences). (B) Activities of these enhancers in the various ps of the visceral mesoderm; black, wild type; red, 24B.GAL4/UAS.Dpp; blue, 24B.GAL4/UAS.Dras1 ${ }^{\text {V12. }}$. Levels of IacZ expression are estimated to be strong, + , or weak, + ; expression trailing into a ps is indicated by ( + ). N ote that BM 1 mediates additional expression in the wild type and in response to ectopic Dpp and Dras $1^{\mathrm{V} 12}$, indicating a constitutive repressor binding to the Mad B sequence. (C) Summary of the results detailed in $B$, implicating the $C R E$ as a response sequence for Ras signaling and $M$ ad $A$ as a response sequence for Dpp signaling (see al so text); +, strong and consistent response; $(+)$ disabled and, in the case of Ras, patchy response; - no response.
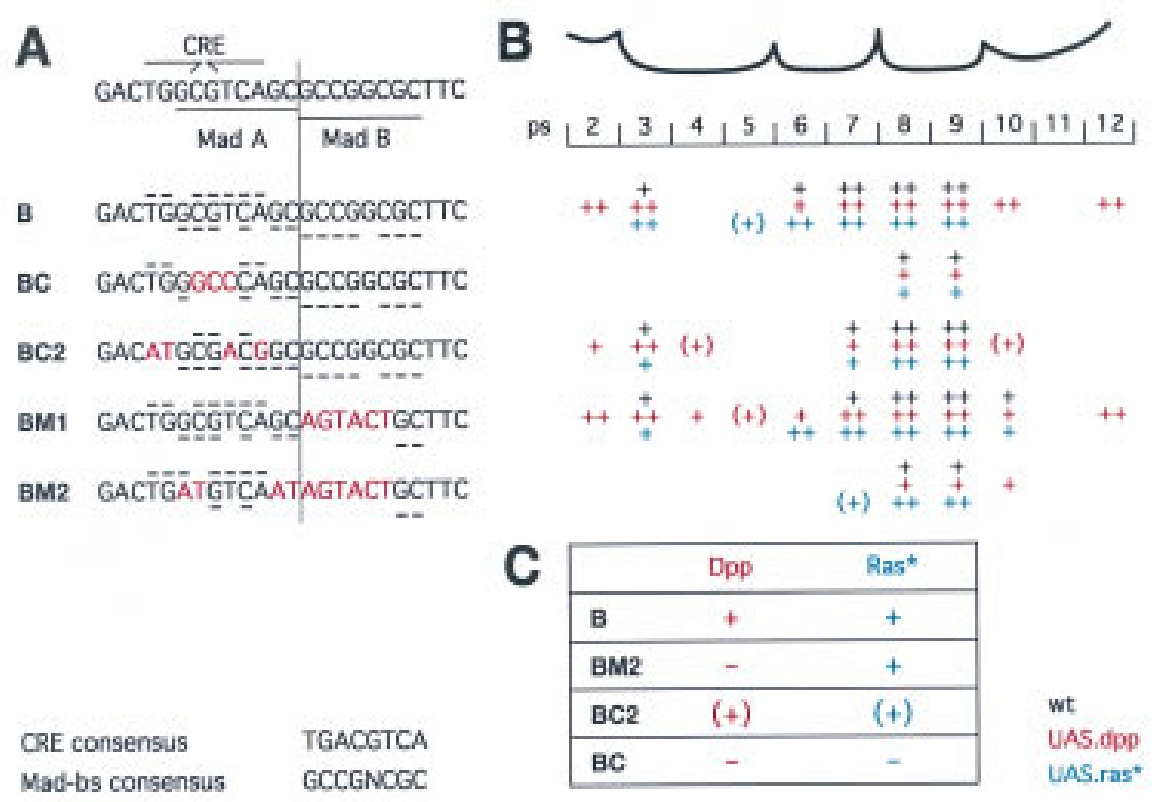

\begin{tabular}{|c|c|c|}
\hline & $D p p$ & Ras* \\
\hline B & + & + \\
\hline BM2 & - & + \\
\hline $\mathrm{BC} 2$ & $(+)$ & $(t)$ \\
\hline $\mathrm{BC}$ & - & - \\
\hline
\end{tabular}

fects of Dpp on endogenous wg (see Staehling-Hampton and Hoffmann 1994). BM 2 still responds well to ectopic $\mathrm{Wg}$ (not shown). BM 2 also responds well to Dras $1^{\mathrm{V} 12}$ by showing increased lacZ staining in ps8 and ps9 as well as conspicuously strong staining in the dorsal mesoderm (Fig. 6l). We conclude that M ad A (or M ad A or Mad B interchangeably) is critical for mediating the $D p p$ response, whereas neither $M$ ad site is required for the response to EGFR signaling.

BC 2 also shows a loss of lacZ staining in ps6 and ps7 of the visceral mesoderm (Fig. 6K), although the effect is

not so drastic as that seen in BM 2 embryos. Staining in ps3 is still moderately strong in BC2. After mesodermal expression of Dpp, we see a limited ectopic response of BC2, mostly in ps2 (Fig. 6J), that corresponds to a region in which the wild-type enhancer is strongly responsive to ectopic D pp (Fig. 5B). However, there is no response of $\mathrm{BC} 2$ to ectopic D pp in the posterior midgut in which the wild-type enhancer is also strongly responsive (Fig. 5B). Evidently, the response of $\mathrm{BC} 2$ to $\mathrm{Dpp}$ is compromised; note that the remaining $D$ pp response of $B C 2$ may reflect its residual CRE activity (as judged by dCREB-B binding;

Figure 6. Responses of wild-type and mutant $\mathrm{U}$ bx enhancers to $\mathrm{Dpp}$ and Dras1 ${ }^{\mathrm{V} 12}$. Side views of 12- to 15-hr-old embryos, bearing $U$ bx $B(A-C)$, BM 1 (D-F), BM $2(G-I)$, or BC2 (J-L), stained with lacZ antibody; (left) 24B.GAL4/UAS.Dpp; (middle) wild type; (right) 24B.GAL4/UAS.Dras1 ${ }^{\mathrm{V} 12}$. Additional staining is seen in response to ectopic Dpp in ps2, in ps9/ps10, and in ps12 (arrowheads in A,D; note that the staining in ps9/ps10 is partly attributable to derepression of endogenous Wg expression; see text); Dras1 ${ }^{\mathrm{v} 12}$ produces increased staining mostly in ps6 but al so in ps8/ps9 (arrowheads in $\mathrm{C}, \mathrm{F}, \mathrm{I})$ and in the dorsal somatic mesoderm (arrows in $\mathrm{C}, \mathrm{I}$; dorsal mesoderm staining is also seen in BM 1 embryos, but this is not visible in the focal plane of $F$ ).

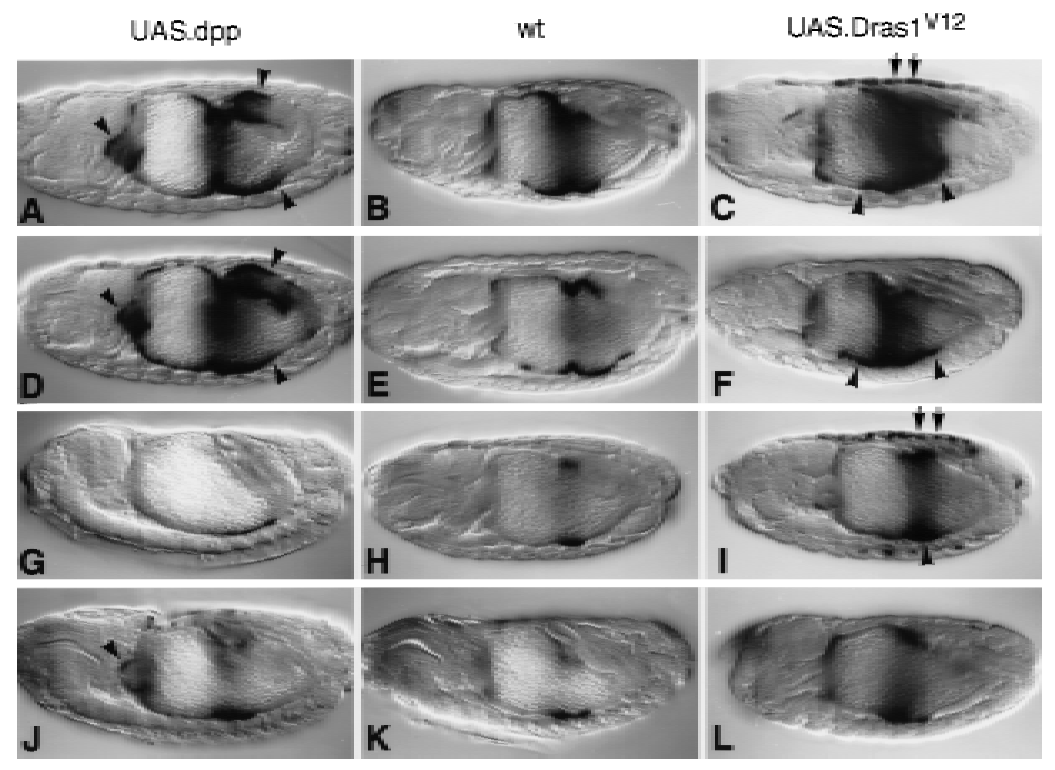


see above). This result strongly suggests that the CRE is critical for the Dpp response, in addition to the $\mathrm{M}$ ad binding sites. It implies that $M$ ad (which still binds to $M$ ad $A$ and $M$ ad $B$ in BC2) does not mediate the Dpp response on its own but depends on interaction with a CRE-binding protein (see below).

The response to Dras ${ }^{\mathrm{V} 12}$ in $\mathrm{BC} 2$ embryos is variable (Fig. 6L): One of the BC2 lines does not show increased lacZ staining (neither in the dorsal somatic nor in the visceral mesoderm), whereas another line shows stimulated lacZ staining in some cells immediately posterior to the incipi ent second constriction (Fig. 6L), and a third line al so shows a limited and patchy stimulation in this region of the midgut as well as some increased staining in the dorsal mesoderm after Dras $1^{\mathrm{V} 12}$ overexpression. Evidently, the Ras response of the BC2 enhancer is neither robust nor consistently detectable and shows some dependence on chromosomal context. Recall that BC2 may retain residual CRE activity (see above) that may explain some of this variability. Whatever the case, the results show that the $\mathrm{BC} 2$ mutation disables the Ras response, pointing to a function of the CRE in the response to EGFR signaling.

Our previously made BC enhancer has base substitutions in the CRE and in $M$ ad $A$; it shows no response to Dpp (Eresh et al. 1997; Fig. 5A,B). We tested this mutant enhancer al so for its response to mesodermal ly expressed Dras $1^{\mathrm{V} 12}$. We found no significant increase in lacZ staining in BC transformants, neither in the midgut nor in the dorsal mesoderm (Fig. 5B); only occasionally, a few mesodermal cells near the middle gut constriction stained more strongly in response to $\operatorname{Dras}^{\mathrm{V} 12}$, a residual response that may be indirect and reflect Ras-stimulated $\mathrm{Dpp}$ or Wg signaling in this region (see above). Given that neither of the $M$ ad sites is required for the response to Ras (as BM 2 responds well to Ras; Fig. 6I), this loss of the Ras response of $B C$ supports our notion that the CRE functions in the response to Ras.

Thus, our mutational dissection of the $U$ bx $B$ enhancer reveals that the $C R E / M$ ad $A$ sequence is a composite response element for Dpp and EGFR signaling. $M$ ad $A$ is critical for the response to $D p p$ but is not necessary for the response to EGFR signaling, whereas the CRE appears to be required for both signals (Fig. 5C). The CRE may medi ate the response to the EGFR component of the Dpp signaling (recall that ectopic Dpp induces ectopic Vein/EGFR signaling). Confirming this notion that the CRE appears to act as an EGFR response element is the observation that a minimal CRE-containing construct (5CRE; Eresh et al. 1997) shows strong synergy (similarly to that of $\mathrm{Ubx} B$; Fig. $4 \mathrm{~J}$ ) in its response to coexpression of Dpp and Dras1 ${ }^{\mathrm{V} 12}$ in the endoderm (not shown).

The Dpp response element in the lab enhancer comprises CREs and Mad binding sites

Our previous work showed that mutation of multiple CREs in the minimal midgut enhancer of lab substantially reduced its activity (Eresh et al. 1997). This en- hancer also contains two Mad binding sites ( $M$ ad A, a low affinity site, and $M$ ad $C$, a high affinity site; Kim et al. 1997); unlike in the U bx enhancer, these Mad binding sites are neither overlapping nor di rectly adjacent to any of the CREs ( $M$ ad $C$ is 51 residues upstream of the tandem CRE2/ 3 sites; cf. Eresh et al. 1997; Kim et al. 1997; Tremml 1991). We asked whether both types of elements are required for the Dpp response of the lab enhancer. Recall that Iab does not respond to ectopic Ras stimulation al one, but bear in mind that its $D p p$ response most likely reflects a combined response to Dpp and EGFR signaling (see above). We thus mutated both $\mathrm{M}$ ad binding sites in the lab enhancer (550M) or both Mad binding sites as well as all 4 CREs (550CM) and compared the activity of these mutant enhancers to that of 550C (all CREs mutated; Eresh et al. 1997) or of the wild-type enhancer (HZ550; Tremml and Bienz 1992) under conditions of normally or ectopically expressed Dpp.

We found that 550M transformants showed only moderately reduced lacZ staining in the endoderm (Fig. 7C) compared with wild-type HZ550 transformants (Fig. 7A) and that their response to ectopic Dpp is moderately, al beit significantly, reduced (Fig. 7, cf. D and B). This indicates that the $\mathrm{M}$ ad binding sites only contribute in a minor way to the Dpp response of the lab enhancer. In contrast, 550C transformants show much reduced lacZ staining compared with HZ550 or 550M transformants (Fig. 7, cf. E, A, and C; see also Eresh et al. 1997) and barely show any additional lacZ staining in response to ectopic D pp (Fig. 7, cf. F, B, and D). The residual response of 550C to ectopic D pp may well reflect a response to $\mathrm{Wg}$ rather than to Dpp/EGFR as the additional staining is seen in a region of the gut (ps8-ps10) where Wg expres-

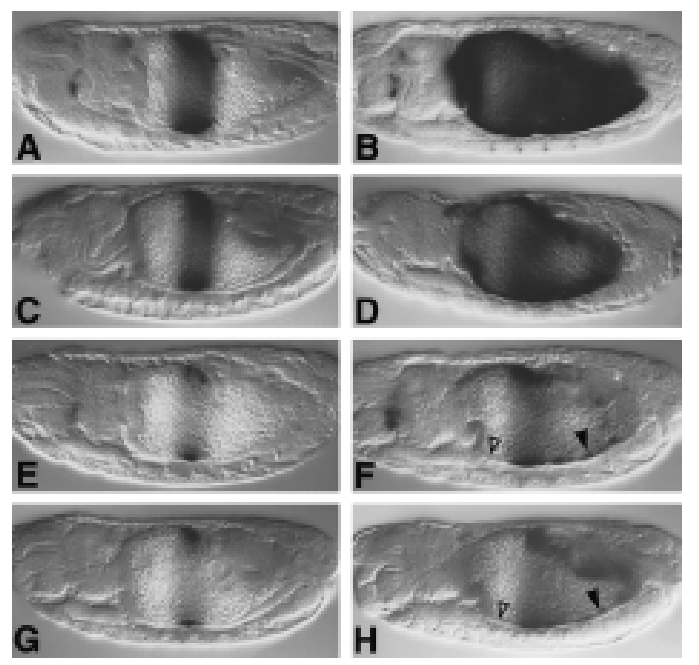

Figure 7. Responses of wild-type and mutant lab enhancers to Dpp. Side views of 12- to 13-hr-old embryos, bearing lab HZ550 $(A, B), 550 M(C, D), 550 C(E, F)$, or $550 C M(G, H)$, stained with lacZ antibody; (left) wild type; (right) 48Y.GAL4/UAS.Dpp. Open triangles in $\mathrm{F}$ and $\mathrm{H}$ indicate complete loss of response to ectopic Dpp in the anterior midgut, whereas arrowheads point to residual response in ps8-ps10 (which may reflect a response to expanded Wg expression; see text). 
sion is expanded in response to ectopic Dpp (StaehlingHampton and Hoffmann 1994; see above). Finally, the lacZ staining patterns in 550CM transformants look similar to those of $550 \mathrm{C}$ transformants (Fig. 7, cf. G and $\mathrm{H}$ with $\mathrm{E}$ and $\mathrm{F}$ ), underscoring the above notion that the $M$ ad binding sites do not play a major role in mediating the Dpp response of the lab enhancer. However, the 550C and 550CM mutant enhancers reveal a critical role of the CREs in this enhancer in its response to Dpp signaling.

These results with the lab enhancer confirm our conclusions derived from the $U$ bx enhancer, namely that the response element to Dpp signaling is bipartite and contains $\mathrm{M}$ ad binding sites as well as CREs. The latter are critical in both cell layers for the signal response, whereas the former seem less criticial in the endoderm than in the visceral mesoderm. Perhaps this reflects the fact that Iab is the ultimate target gene of the endoderm induction (Bienz 1997) and that its enhancer clearly integrates a number of distinct positional inputs (Tremml and Bienz 1992; Grieder et al. 1997), some of which may be partially redundant.

\section{Discussion}

An inductive process subdivides the endoderm of Drosophila along its axis into sections with distinct cell types. This induction is mediated by Dpp and Wg, secreted from localized sources in the visceral mesoderm, which synergi ze during this process to confer anteroposterior position (Bienz 1997; Fig. 1). Here, we report the discovery of Vein as a secondary signal of Dpp and Wg. Vein is up-regulated in the visceral mesoderm in response to Dpp and $\mathrm{Wg}$ and, according to our evidence, signals through Drosophila EGFR and Ras to both cell Iayers of the embryonic midgut. Like Dpp and Wg, Vein/ EGFR signaling plays an important role in the control of key homeotic genes in these cell layers, in the parautocrine loop of $\mathrm{Ubx}$ in the visceral mesoderm, and in the induction of lab in the subjacent endoderm (Fig. 8).

\section{Vein, the ligand stimulating EGFR during endoderm induction}

The following evidence indi cates that Vein is the ligand stimulating EGFR in the visceral mesoderm and endoderm. First, vein mutant embryos closely resemble flb mutants (lacking EGFR function) or embryos in which EGFR function is blocked in either cell layer of the midgut by targeted expression of DN-DER. Second, vein is expressed at high levels in localized sources in the midgut that correspond roughly to the regions in which the main defects of lack of EGFR function are observed.

Although vein has been shown to contribute to EGFR activity in the larval and imaginal epi dermis (Schnepp et al. 1996; Simcox et al. 1996; Simcox 1997), the main EGFR ligand in most epidermal tissues appears to be Spitz (Rutledge et al. 1992; Freeman 1994, 1996; Schweitzer et al. 1995a; O'Keefe et al . 1997; Szüts et al. 1997). spitz expression is fairly ubiquitous (Rutledge et

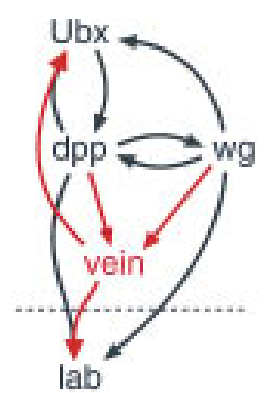

Figure 8. Vein, a secondary signal in the inductive cascade: summary. Vein, the activating ligand of EGFR in the midgut, is a secondary signal whose expression is up-regulated by $D p p$ and Wg. Vein/EGFR signaling is required for lab induction in the endoderm (bel ow broken line) and stimulates $U$ bx expression in the visceral mesoderm (above broken line). Its functional intertwining with Dpp signaling is indicated by merged arrows. Other stimulatory interactions between signaling pathways and responding genes are given by black arrows (most of these interactions are known to be direct, based on physical evidence; see text).

al. 1992), but localized sources of active Spitz ligand appear to be generated by localized expression of the membrane-spanning protein Rhomboid (Bier et al. 1990; Freeman 1994; Schweitzer et al . 1995a; Golembo et al. 1996a; Gabay et al. 1997). Our analysis of spitz and rhomboid mutants shows that these genes do not have a major role during endoderm induction. They do, however, show minor effects on lab induction, and rhomboid appears to be expressed at low levels in bands spanning each of the three midgut constrictions (see $M$ aterials and M ethods), suggesting that there may be localized sources of low levels of active Spitz near these constrictions. Occasional vein mutant larvae hatch (Schnepp et al. 1996; D. Szüts, S. Eresh, and M. Bienz, unpubl.); these may escape embryonic death because Rhomboid and Spitz back up some of the Vein function.

Our mutant analysis suggests strongly that Vein is the main, if not the only, ligand that stimulates EGFR in the embryonic midgut. This contrasts with other tissues, mainly of ectodermal origin, in which Spitz is the main EGFR ligand (see below). Interestingly, Vein al so has a major role during an inductive process between muscle and epidermis: Vein is secreted from muscle cells and triggers differentiation of the receiving epidermal cells into tendon cells (Yarnitzky et al. 1997). These functions of Vein during inductive processes between different cell layers suggest that the molecular properties of Vein are particularly suited to such processes that require the signal to cross basal membranes. Similarly, the extensive mesodermal expression of Vein may mean that this signal protein is particularly well-adapted to its production in this cell layer. N ote that Vein is similar to mammalian neuregulins (Schnepp et al. 1996) that appear to function in developmental contexts that involve communication between different cell layers (Meyer and Birchmeier 1995; for review, see Lemke 1996).

We have not seen any midgut defects in argos mutant embryos. Either there is no function of this inhibitory 
ligand of EGFR (Schweitzer et al. 1995b) in the midgut, or we have missed such a function: argos function in other tissues can be subtle (e.g., Szüts et al. 1997), and the midgut may lack the cellular markers necessary to reveal such a subtle function. Also, argos mutation is tantamount to EGFR overactivation (Schweitzer et al. 1995b), and our results with Dras $1^{\mathrm{V} 12}$ showed that the effects of EGFR overactivation can be hard to detect in the midgut because of the permissive role of EGFR signaling in this tissue.

\section{Intertwining of Dpp and EGFR signaling functions}

Vein/EGFR signaling has a permissive role during endoderm induction in that it appears to be effective only in the presence of Dpp signaling. The converse is also true: Dpp signaling is only effective with concomitant Vein/ EGFR signaling. Dpp up-regulates Vein expression in its own real $m$ of action and thus stimulates its own partner signal, thereby acquiring an apparent instructive role in conferring position during endoderm induction. D pp signaling in the midgut must therefore be viewed as bipartite, consisting of Dpp signaling proper and of secondary Vein/EGFR signaling.

This bipartite nature of the Dpp signal is reflected by the two types of D pp target sequences in the $U$ bx and I $a b$ enhancers, the CREs and the $M$ ad binding sites. As these overlap in the case of $U$ bx, they were identified previously as a single target (Eresh et al. 1997), but our current analysis illustrates very clearly that the Dpp response element is bipartite in both enhancers. Our evidence suggests that mutation of the $\mathrm{M}$ ad binding sites affects exclusively the response to Dpp, whereas mutation of the CRE affects the response to both Dpp and EGFR. In the case of the lab enhancer, the CREs are more critical for the Dpp response than the Mad binding sites. This parallels the layout of a mammalian enhancer (Yingling et al. 1997) in which a TGF- $\beta$ response element comprises a Smad3/Smad4 binding site as well as an AP1 binding site (an element related to the $C R E$ ); in this case too, the AP1 binding site is critical for the signal response, whereas the Smad binding site is not. N ote that our analysis leaves open the possi bility that there may be additional target sites for EGFR signaling. Also, in the case of the lab enhancer, it was demonstrated that the Dpp response also depends on another enhancer element, distinct from CREs and Mad binding sites, that mediates additional positional inputs (Grieder et al. 1997).

As al ready pointed out, Dpp signaling may achieve its functional autonomy only because it activates expression of its own partner signal. This may imply that $\mathrm{Mad}$ and other Smads, despite their containing a conserved transcriptional activation domain (Liu et al. 1996), do not act autonomously in stimulating Dpp target genes but require functional interaction with other enhancer binding factors. In the case of our two midgut enhancers, its partner factor is likely to be a protein binding to the CRE. As al ready mentioned, a partner factor for mammalian Smads appears to be AP1 (Yingling et al. 1997), a protein factor related to CREBs. A distinct candidate for a Mad partner is Schnurri, a protein with similarity to a family of mammalian transcription factors that is required downstream of the Dpp signal (Arora et al. 1995; Grieder et al. 1995). Finally, in Xenopus embryos, a protein called Fast- 1 has been identified that binds to an activin-responsive element in an activin target gene (Huang et al. 1995) and with which Smads associate on activin signaling (Chen et al. 1996, 1997). Whether or not these $\mathrm{M}$ ad/Smad partners themselves are targets for distinct signaling pathways, as the U bx CRE-binding protein appears to be, is an open question. Whatever the case, their existence suggests that the Dpp signaling pathway itself is not autonomous in conferring position but needs close cooperation with other positional inputs.

\section{Why a secondary signal?}

A number of secondary signals have been described during Drosophila development whose localized expression is activated by a primary signal, and Dpp and EGFR signaling can be involved in such primary-secondary signal relationships. In many developmental contexts, the secondary signal is thought to function entirely to relay the effects of the primary signal (e.g., Basler and Struhl 1994; Capdevila and Guerrero 1994; Glise and Noselli 1997; Hou et al. 1997; O'Keefe et al. 1997; Szüts et al. 1997; Riesgo-Escovar and Hafen 1997). But there are indications in one of these cases that primary and secondary signals may cooperate in implementing their effects (Glise and Noselli 1997). Finally, Argos is a secondary signal that clearly impinges on the function of the primary signal Spitz (Freeman 1996; Golembo et al. 1996b).

The embryonic midgut in which EGFR seconds Dpp signal ing is perhaps the clearest example as yet of a situation where the functions of the two signals are intertwined and interdependent. M ore remarkably, the source of the secondary signal in this case is coextensive with that of the primary signal; because of this, the secondary signal confers an apparent autonomy on the primary signal in conveying position. Why should there be this secondary signal whose role is entirely permissive, namely to assist the primary signal in implementing its tasks? We can think of two kinds of answers.

The first one is based on the observation that lack of Vein/EGFR signaling in the midgut appears to make cells sick and perhaps causes them to die. Therefore, Vein/EGFR signaling may serve as a "survival signal". Intriguingly, cell survival in embryos lacking vein or EGFR function appears to be affected preferentially near the two Dpp sources (where vein expression is up-regulated). Perhaps, high levels of Dpp signaling can cause cell death, and vein signaling may be up-regulated to counteract a putative local deleterious effect of Dpp. A precedent for such a scenario may be found in the developing chick limb bud where the cell death-inducing properties of BMP (a TGF- $\beta$-like signal) seem to be antagonized locally by a signal triggering the Ras pathway (Gañan et al. 1996). However, although antagonistic effects between EGFR- and TGF- $\beta$-type signaling have 
been observed (e.g., Kretschmar et al. 1997; Wappner et al. 1997), our evidence suggests strongly that Vein/EGFR and Dpp both act positively in the embryonic midgut of Drosophila. Furthermore, they synergize with each other in the transcriptional stimulation of target genes. This observed synergy parallels cooperation between Ras and TGF- $\beta$ signaling during epithelial tumor progression (e.g., Oft et al. 1996 and references therein). We therefore think it unlikely that Vein functions in the midgut entirely as a survival signal near Dpp sources.

The second kind of answer builds on our observations that indicate functional interdependence and synergy of the two signaling pathways in stimulating transcription of target genes. This could be beneficial for developmental systems in two ways: First, if cells need to be costimulated by cooperating primary and secondary signals, this would serve to sharpen their signal response. This putative sharpening effect may be a contributory factor in sharp responses to signaling thresholds such as those observed in the Xenopus embryo (Green et al. 1992). Second, the need for costimulation would safeguard against fortuitous and random stimulation of cells by any one signal, thus improving the reliability of their signal response. And although we have observed a requirement for the secondary signal throughout the functional real $m$ of the primary signal, we envisage that the role of the secondary signal is particularly critical in remote cells where the distribution of the primary signal becomes shallow, imprecise, and unreliable. Therefore, the secondary signal may provide primarily "remote stimulation" (see al so Freeman 1996).

Whatever the case, it seems very likely that the use of a functionally coupled primary-secondary signal system results in a refinement and stabilization of positional information and in a degree of precision of this information that could not be conferred by one signal alone. Functional intertwining of a secondary and a primary signal may represent a mechanistic solution of how morphogens such as D pp and activins work. Perhaps, signal ing pathways do not function on their own in eliciting multiple different cellular responses, as envisaged by the purest version of the morphogen concept.

\section{Materials and methods}

Fly strains

The following mutant alleles were used: $\mathrm{dpp}^{\mathrm{s4}}$ (Immerglück et al. 1990), wg ${ }^{\prime L 114}$ (N üsslein-Volhard et al. 1984), flb ${ }^{1 K 35}$ (null allele; N üsslein-Volhard et al. 1984), flb ${ }^{1 F 26}$ (temperature-sensitive allele; Clifford and Schüpbach 1992), and aos ${ }^{1 \Delta 7}$ (Freeman et al. 1992). Of the two rhomboid alleles used, only rho ${ }^{7 \mathrm{M} 43}$ homozygotes (M ayer and N üssl ein-Vol hard 1988) showed the midgut phenotype described in the text, whereas rho ${ }^{\mathrm{P} \Delta 5}$ homozygotes have normal guts. Three vein alleles were used: $\mathrm{vn}^{\gamma 3}, \mathrm{vn}^{\gamma 4}$ (Simcox et al. 1996), and $\mathrm{vn}^{\mathrm{P} 1749}$ (a P-element insertion into vein; Yarnitzky et al. 1997); the null allele vn ${ }^{\gamma 4}$ showed the strongest midgut phenotype (homozygously or in transheterozygotes with $\mathrm{vn}^{\gamma 3}$ ), whereas $\mathrm{vn}^{\gamma 3}$ homozygotes showed a slightly weaker phenotype; $\mathrm{vn}^{\mathrm{P} 1749}$ homozygotes showed a very mild phenotype, with Lab staining missing only in a few endodermal cells, but $\mathrm{vn}^{\mathrm{P} 1749} / \mathrm{vn}^{\gamma 4}$ and $\mathrm{vn}^{\mathrm{P} 1749} / \mathrm{vn}^{\gamma 3}$ transheterozy- gotes showed a moderately strong midgut phenotype (the former slightly stronger than the latter; Fig. 2G; data not shown). M utant embryos lacking Spitz function were produced from homozygous mutant germ-line clones (Chou et al. 1993), using spi ${ }^{\mathrm{SE1}}$ or spi ${ }^{\mathrm{A} 14}$ on an FRT 40A chromosome (Tio et al. 1994); midguts of paternally rescued embryos were normal, whereas those of embryos without zygotic Spitz function showed the mild phenotype described in Results.

The following GAL4 driver and responder lines were used: 24B.GAL4 (Brand and Perrimon 1993), 48Y.GAL4 (M artin-Bermudo et al. 1997), UAS.Dpp (Staehling-Hampton and Hoffmann 1994), UAS.Wg (Lawrence et al. 1996), UAS.DN-DER (O'Keefe et al. 1997), UAS.Vein (Schnepp et al. 1996), and UAS.Dras1 ${ }^{\mathrm{V} 12}$ (Lee et al. 1996).

lacZ reporter lines bearing $U$ bx $B$ (Bhz; Thüringer and Bienz 1993), BC (Eresh et al. 1997), lab HZ550, and 550C (T remml and Bienz 1992; Eresh et al. 1997) have been described. The enhancer trap line A490 (Hartenstein and Jan 1992) was used to visualize endodermal cells. The enhancer trap line rholac1 (Bier et al. 1990) showed weak lacZ staining in the visceral mesoderm, mostly noticeable around the midgut constrictions, indicating rhomboid expression at these sites.

Phenotypic analysis

Standard crosses were set up, and embryos were collected at $25^{\circ} \mathrm{C}$ (unless specified otherwise). Homozygous mutant embryos were either identified by their midgut phenotypes or by the use of lacZ-bearing bal ancer chromosomes.

In most crosses involving UAS constructs, embryos were collected for $6-8 \mathrm{hr}$ at $25^{\circ} \mathrm{C}$ and subsequently aged at $18^{\circ} \mathrm{C}$ until fixation (note that the mutant phenotypes are usually weaker after ageing the embryos at low temperature, owing to lowered transgene expression under these conditions). Embryos overexpressing Dpp, Wg, or DN-DER from UAS constructs were readily identified by their mutant phenotypes, whereas embryos expressing UAS.Dras1 ${ }^{\mathrm{V} 12}$ and $U \mathrm{bx}$ reporter constructs were identified by their characteristic lacZ staining in the dorsal somatic mesoderm (this identification was confirmed by the use of lacZ balancer chromosomes). For coexpression of Dpp and DN-DER or Dras1 ${ }^{\mathrm{V} 12}$, recombinant chromosomes bearing the two respective UAS transposons were generated.

For the analysis of temperature-sensitive alleles, the following conditions were used: To remove EGFR function before midgut formation, but after germ-band retraction, $\mathrm{flb}^{1 \mathrm{~F} 26} \mathrm{mu}-$ tant embryos (collected for $1 \mathrm{hr}$ at $18^{\circ} \mathrm{C}$ ) were shifted to the restrictive temperature $\left(29^{\circ} \mathrm{C}\right)$ after $12 \mathrm{hr}$ at $18^{\circ} \mathrm{C}$. To remove $\mathrm{Wg}$ function before midgut formation, $\mathrm{wg}^{\mathrm{L} L 114}$ embryos (collected for $1 \mathrm{hr}$ at $18^{\circ} \mathrm{C}$ ) were shifted to the restrictive temperature $\left(25^{\circ} \mathrm{C}\right)$ after $24 \mathrm{hr}$ at $15^{\circ} \mathrm{C}$.

Antibodies against the following proteins were used: LacZ (Promega), Lab (Riese et al. 1997a), Ubx (White and Wilcox 1984), Dpp (Panganiban et al. 1990), and Wg (Brook and Cohen 1996). Staining of embryos was done as described (Szüts et al. 1997).

vein expression was detected by in situ hybridization, using DIG-labeled single-stranded DNA probes generated by asymmetric PCR (see al so Schnepp et al. 1996). A probe equival ent to probe B of Simcox et al. (1996; Fig. 1) was mostly used, but a shorter probe spanning the protein-coding exon 2-4 (Schnepp et al. 1996) gave an identical expression pattern.

\section{P-element transformation}

Mutant $\mathrm{U}$ bx and lab constructs were generated by the introduction of base substitutions (Fig. 5A) into the wild-type $U$ bx B 
or lab HZ550 enhancers (see Eresh et al. 1997). The following mutator oligonucleotides were used to mutagenize the $\mathrm{Mad}$ binding sites in HZ550 (mutagenized resi dues in small letters): GACAGATACGGGagtaCtTGGGGAGACACC (Mad A) and GAATCGTATCGAACtcgaGaACTCCAAGTTCC (M ad C). For each construct, several independent transformant lines were isol ated and tested for their response to ectopic D pp or D $\operatorname{~ras}^{\mathrm{V} 12}$ as described (Eresh et al. 1997). In these experiments, embryos were usually kept at $25^{\circ} \mathrm{C}$ (except for the synergy experiments in which they were aged at $18^{\circ} \mathrm{C}$, as described above), to visualize a maximal response to $\mathrm{Dpp}$ or Dras $1^{\mathrm{V} 12}$.

\section{In vitro binding assays}

Mutant enhancers were tested for their ability to bind $M$ ad and CREB-B as follows: An amino-terminal fragment of M ad (GST$\mathrm{N}$ mad, a generous gift of L. Waltzer (M RC); see Kim et al. 1997) was expressed as a GST fusion protein in bacteria, and crude extracts were prepared and used for in vitro binding assays and subsequent analysis by mobility retardation assays as described (Eresh et al. 1997). DNA binding of GST-N mad or CREB-B (Eresh et al. 1997) was monitored with radioactively labeled wild-type and mutant oligomers (23 nucleotides long) spanning the MadA/CRE sequence in the $\mathrm{Ubx}$ enhancer (Fig. 5A); the same oligomers were also used in competition assays with the wild-type oligomer as a labeled probe as described (Eresh et al. 1997). We found that GST-N mad binds to M ad A and M ad B with comparable affinity, indicating that the error in the original M ad A sequence [used by Kim et al. (1997) to measure DN Abinding affinity] led to an underestimate of Mad's affinity to M ad A (for correction of sequence, see Eresh et al. 1997). Our binding experiments al so confirmed that the base substitutions in BM 1 and BM 2 abolished sequence-specific binding of GST$\mathrm{N}$ mad, whereas those in BC2 did not affect it. In the case of dCREB-B, we found that the base substitutions in BM 1 did not affect its affinity to the $C R E$, but those in $B M 2$ reduced it by a factor of 9, whereas those in BC2 reduced it by a factor of 20; binding of dCREB-B to BC was undetectable (i.e., reduced it by a factor of $>100$ ), as reported (Eresh et al. 1997). Values represent averages from three different experiments. These binding assays indicate reduced CRE function in BM 2 but also residual $C R E$ function in $\mathrm{BC} 2$.

\section{Acknowledgments}

We thank Mandy Simcox for sending us embryos hybridized with vein probes for initial examination and for providing vein DNA. We are also grateful to Erez Raz and Benny Shilo for providing the UAS.DN-DER strain before publication and to Allen Laughon for sharing unpublished results and advice on DNA binding of Mad. We further thank Lucas Waltzer for the Mad expression plasmid, Matthew Freeman for various fly strains and for discussion, and Xiang $\mathrm{Yu}$ and Lucas Waltzer for comments on the manuscript. D.S. is supported by a studentship from Trinity College, Cambridge.

The publication costs of this article were defrayed in part by payment of page charges. This article must therefore be hereby marked "advertisement" in accordance with 18 USC section 1734 solely to indicate this fact.

\section{References}

Arora, K., H. Dai, S.G. Kazuko, J. Jamal, M.B. O'Connor, A. Letsou, and R. Warrior. 1995. The Drosophila schnurri gene acts in the Dpp/TGF- $\beta$ signaling pathway and encodes a transcription factor homologous to the human M BP family. Cell 81: 781-790.

Basler, K. and G. Struhl. 1994. Compartment boundaries and the control of Drosophila limb pattern by Hedgehog protein. Nature 368: 208-214.

Bienz, M. 1997. Endoderm induction in Drosophila: The nuclear targets of the inducing signals. Curr. Opin. Genet. Dev. 7: 683-688.

Bier, E., L.Y. Jan, and Y.N . Jan. 1990. rhomboid, a gene required for dorsoventral axis establishment and peripheral nervous system development in Drosophila melanogaster. Genes \& Dev. 4: 190-203.

Brand, A.H. and N . Perrimon. 1993. Targeted gene expression as a means of altering cell fates and generating dominant phenotypes. Development 118: 401-415.

Brook, V.J. and S. Cohen. 1996. Antagonistic interactions between Wingless and Decapentaplegic responsible for dorsalventral pattern in the Drosophila leg. Science 273: 13721377.

Campos-Ortega, J.A. and V. Hartenstein. 1985. The embryonic development of Drosophila melanogaster. Springer Verlag, New York, NY.

Capdevila, J. and I. Guerrero. 1994. Targeted expression of the signaling mol ecule decapentapl egic induces pattern duplications and growth alterations in Drosophila wings. EMBO J. 13: 4459-4468.

Chen, X., M.J. Rubock, and M. Whitman. 1996. A transcriptional partner for MAD proteins in TGF- $\beta$ signaling. Nature 383: 691-696.

Chen, X., E. Weisberg, V. Fridmacher, M. Watanabe, G. N aco, and M. Whitman. 1997. Smad4 and FAST -1 in the assembly of activin-responsive factor. Nature 389: 85-89.

Chou, T.-B., E. Noll, and N. Perrimon. 1993. Autosomal P[o$\mathrm{vo}^{\mathrm{D} 1}$ ] dominant female-sterile insertions in Drosophila and their use in generating germ-line chimeras. Development 119: 1359-1369.

Clifford, R. and T. Schüpbach. 1992. The torpedo (DER) receptor tyrosine kinase is required at multiple times during Drosophila embryogenesis. Development 115: 853-872.

de Groot, R.P., J. den Hertog, J.R. Vandenheede, J. Goris, and P. Sassone-Corsi. 1993. Multiple and cooperative phosphorylation events regulate the CREM activator function. EMBO J. 12: 3903-3911.

Eresh, S., J. Riese, D.B. Jackson, D. Bohmann, and M. Bienz. 1997. A CREB binding site as a target for decapentaplegic signaling during Drosophila endoderm induction. EMBO J. 16: 2014-2022.

Ferguson, E.L. and K.V. Anderson. 1992. decapentaplegic acts as a morphogen to organize dorsal-ventral pattern in the Drosophila embryo. Cell 71: 451-461.

Freeman, M. 1994. The spitz gene is required for photoreceptor determination in the Drosophila eye where it interacts with the EGF receptor. Mech. Dev. 48: 25-33.

- - . 1996. Reiterative use of the EGF receptor triggers differentiation of all cell types in the Drosophila eye. Cell 87: $651-660$.

Freeman, M., C. Klämbt, C.S. Goodman, and G.M. Rubin. 1992. The argos gene encodes a diffusible factor that regulates cell fate decisions in the Drosophila eye. Cell 69: 963-975.

Gabay, L., R. Seger, and B.-Z. Shilo. 1997. In situ activation pattern of Drosophila EGF receptor pathway during development. Science 277: 1103-1106.

Gañan, Y., D. Macias, M. Duterque-Coquillaud, M.A. Ros, and J.M. Hurle. 1996. Role of TGF- $\beta$ s and BM Ps as signals controlling the position of the digits and the areas of interdigital cell death in the developing chick limb autopod. Develop- 
ment 122: 2349-2357.

Ginty, D.D., A. Bonni, and M .E. Greenberg. 1994. N erve growth factor activates a Ras-dependent protein kinase that stimulates c-fos transcription via phosphorylation of CREB. Cell 77: 713-725.

Glise, B. and S. N oselli. 1997. Coupling of Jun amino-terminal kinase and Decapentaplegic signaling pathways in Drosophila morphogenesis. Genes \& Dev. 11: 1738-1747.

Golembo, M., E. Raz, and B.-Z. Shilo. 1996a. The Drosophila embryonic midline is the site of Spitz processing, and induces activation of the EGF receptor in the ventral ectoderm. Development 122: 3363-3370.

Golembo, M., R. Schweitzer, M. Freeman, and B.-Z. Shilo. 1996b. argos transcription is induced by the Drosophila EGF receptor pathway to form an inhibitory feedback loop. De velopment 122: 223-230.

Green, J.B.A. and J.C. Smith. 1990. Graded changes in dose of a Xenopus activin A homologue elicit stepwise transitions in embryonic cell fate. Nature 347: 391-394.

Green, J.B.A., H.V. New, and J.C. Smith. 1992. Responses of embryonic Xenopus cells to activin and FGF are separated by multiple dose thresholds and correspond to distinct axes of the mesoderm. Cell 71: 731-739.

Grieder, N.C., D. N ellen, R. Burke, K. Basler, and M. Affolter. 1995. Schnurri is required for Drosophila Dpp signaling and encodes a zinc finger protein similar to the mammalian transcription factor PRDII-BF1. Cell 81: 791-800.

Grieder, N.C., T. Marty, H.-D. Ryoo, R.S. Mann, and M. Affolter. 1997. Synergistic activation of a Drosophila enhancer by HOM / EXD and DPP signaling. EMBO J. 16: 7402-7410.

Gurdon, J.B., P. Harger, A. Mitchell, and P. Lemaire. 1994. ACtivin signalling and response to a morphogen gradient. Nature 371: 487-492.

Hartenstein, V. and Y.N. Jan. 1992. Studying Drosophila embryogenesis with P-LacZ enhancer trap lines. Wilhelm Roux's Arch. Dev. Biol. 201: 194-220.

Hemmati-Brivanlou, A. and D.A. Melton. 1992. Truncated activin receptor inhibits mesoderm induction and formation of axial structures in Xenopus embryos. Nature 359: 609-614.

Hoppler, S. and M. Bienz. 1994. Specification of a single cell type by a Drosophila homeotic gene. Cell 76: 689-702.

- - - 1995. Two different thresholds of wingless signaling with distinct developmental consequences in the Drosophila midgut. EMBO J. 14: 5016-5026.

Hou, X.S., E.S. Goldstein, and N. Perrimon. 1997. Drosophila Jun relays the Jun amino-terminal kinase signal transduction pathway to the Decapentaplegic signal transduction pathway in regulating epithelial cell sheet movement. Genes \& Dev. 11: 1728-1737.

Huang, H.-C., L.C. Murtaugh, P.D. Vize, and M. Whitman. 1995. Identification of a potential regulator of early transcriptional responses to mesoderm inducers in the frog embryo. EMBO J. 14: 5965-5973.

Hursh, D.A., R.W. Padgett, and W.M. Gel bart. 1993. Cross reguIation of decapentaplegic and U Itrabithorax transcription in the embryonic visceral mesoderm of Drosophila. Development 117: 1211-1222.

Immerglück, K., P.A. Lawrence, and M. Bienz. 1990. Induction across germ layers in Drosophila mediated by a genetic cascade. Cell 62: 261-268.

Irish, V.F. and W.M. Gel bart. 1987. The decapentapl egic gene is required for dorsal-ventral patterning of the Drosophila embryo. Genes \& Dev. 1: 868-879.

Kessler, D. and D.A. Melton. 1995. Induction of dorsal mesoderm by soluble, mature Vgl protein. Development 121: $2155-2164$.
Kim, J., K. Johnson, H.J. Chen, S. Carroll, and A. Laughon. 1997. MAD binds to DNA and directly mediates activation of vestigial by DPP. Nature 388: 304-308.

Kretschmar, M., J. Doody, and J. Massagué. 1997. Opposing BMP and EGF signaling pathways converge on the TGF- $\beta$ family mediator Smad1. Nature 389: 618-622.

Lawrence, P.A., B. Sanson, and J.-P. Vincent. 1996. Compartments, wingless and engrailed: Patterning the ventral epi dermis of Drosophila embryos. Development 122: 4095-4103.

Lawrence, P.A. and G. Struhl. 1996. Morphogens, compartments, and patterns: Lessons from Drosophila? Cell 85: 951961.

Lecuit, T., W.J. Brook, M. N g, M. Calleja, H. Sun, and S.M. Cohen. 1996. Two distinct mechanisms for long-range patterning by Decapentaplegic in the Drosophila wing. Nature 381: 387-393.

Lee, T., L. Feig, and D.J. Montell. 1996. Two distinct roles for Ras in a developmentally regulated cell migration. Development 122: 409-418.

Lemke, G. 1996. N euregulins in development. Mol. Cell. Neurosci. 7: 247-262.

Liu, F., A. Hata, J.C. Baker, J. Doody, J. Cárcamo, R.M. Harland, and J. Massagué. 1996. A human Mad protein acting as a BM P-regulated transcriptional activator. Nature 381: 620623.

Martin-Bermudo, M.D., O.M. Dunin-Borkowski, and N.H. Brown. 1997. Specificity of PS integrin function during embryogenesis resides in the $\alpha$ subunit extracellular domain. EMBO J. 16: 4184-4193.

Massagué, J., A. Hata, and F. Liu. 1997. TGF- $\beta$ signaling through the Smad pathway. Trends Cell Biol. 7: 187-192.

Mayer, U. and C. N üsslein-Volhard. 1988. A group of genes required for pattern formation in the ventral ectoderm of the Drosophila embryo. Genes \& Dev. 2: 1496-1511.

Meyer, D. and C. Birchmeier. 1995. Multiple essential functions of neuregulin in development. Nature 378: 386-390.

Montminy, M.R., K.A. Sevarino, J.A. Wagner, G. Mandel, and R.H. Goodman. 1986. Identification of a cyclic-AM P-responsive element within the rat somatostatin gene. Proc. Natl. Acad. Sci. 83: 6682-6686.

Nellen, D., R. Burke, G. Struhl, and K. Basler. 1996. Direct and long-range action of a DPP morphogen gradient. Cell 85: 357-368.

N euman-Sil berberg, F. and T. Schüpbach. 1993. The Drosophila gene gurken produces a dorsally local ized RN A and encodes a TGF $\alpha$-like protein. Cell 75: 165-174.

N ewfeld, S.J., E.H. Chartoff, J.M. Graff, D.A. M elton, and W.M. Gel bart. 1996. Mothers against dpp encodes a conserved cytoplasmic protein required in DPP/TGF- $\beta$ responsive cells. Development 122: 2099-2108.

N üsslein-Volhard, C., E. Wieschaus, and H. Kluding. 1984. M utations affecting the pattern of the larval cuticle in Drosophila melanogaster.-I. Zygotic loci on the second chromosome. Wilhelm Roux's Arch. Dev. Biol. 193: 267-282.

Oft, M., J. Peli, C. Rudaz, H. Schwarz, H. Beug, and E. Reichmann. 1996. TGF- $\beta 1$ and Ha-Ras collaborate in modulating the phenotypic plasticity and invasiveness of epithelial tumor cells. Genes \& Dev. 10: 2462-2477.

O'Keefe, L., S.T. Dougan, L. Gabay, E. Raz, B.-Z. Shilo, and S. DiN ardo. 1997. Spitz and Wingless, emanating from distinct borders, cooperate to establish cell fate across the Engrailed domain in the Drosophila epidermis. Development 124: 4837-4845.

Panganiban, G.E.F., R. Reuter, M.P. Scott, and F.M. Hoffmann. 1990. A Drosophila growth factor homolog, decapentaplegic, regulates homeotic gene expression within and across 
germ layers during midgut morphogenesis. Development 110: 1041-1050.

Raz, E. and B.-Z. Shilo. 1992. Dissection of the faint little ball (flb) phenotype: Determination of the development of the Drosophila central nervous system by early interactions in the ectoderm. Development 114: 113-123.

Reuter, R., G.E.F. Panganiban, F.M. Hoffmann, and M.P. Scott. 1990. Homeotic genes regulate the expression of putative growth factors in the visceral mesoderm of Drosophila embryos. Development 110: 1031-1040.

Riese, J., G. Tremml, and M. Bienz. 1997a. D-Fos, a target gene for Decapentaplegic signalling with a critical role during Drosophila endoderm induction. Development 124: 33533361.

Riese, J., X. Yu, A. M unnerlyn, S. Eresh, R. Grosschedl, and M. Bienz. 1997b. LEF-1, a nuclear factor coordinating signaling inputs from wingless and decapentaplegic. Cell 88: 777-787.

Riesgo-Escovar, J.R. and E. Hafen. 1997. Drosophila Jun kinase regulates expression of decapentaplegic via the ETS-domain protein Aop and the AP-1 transcription factor DJun during dorsal closure. Genes \& Dev. 11: 1717-1727.

Rutledge, B.J., K. Zhang, E. Bier, Y.N. Jan, and N. Perrimon. 1992. The Drosophila spitz gene encodes a putative EGF-like growth factor involved in dorsal-ventral axis formation and neurogenesis. Genes \& Dev. 6: 1503-1517.

Saari, G. and M. Bienz. 1987. The structure of the U Itrabithorax promoter of Drosophila melanogaster. EMBO J. 6: 17751779.

Schnepp, B., G. Grumbling, T. Donal dson, and A. Simcox. 1996. Vein is a novel component in the Drosophila epidermal growth factor receptor pathway with similarity to the neuregulins. Genes \& Dev. 10: 2302-2313.

Schweitzer, R., M. Shaharabany, M. Seger, and B.-Z. Shilo. 1995a. Secreted Spitz triggers the DER signalling pathway and is a limiting component in embryonic ventral ectoderm determination. Genes \& Dev. 9: 1518-1529.

Schweitzer, R., R. Howes, R. Smith, B.-Z. Shilo, and M. Free man. 1995b. Inhibition of Drosophila EGF receptor activation by the secreted protein Argos. Nature 376: 699-702.

Sekelsky, J., S. N ewfeld, L. Raftery, E. Chartoff, and W.M. Gelbart. 1995. Genetic characterization and cloning of Mothers against dpp, a gene required for decapentaplegic function in Drosophila melanogaster. Genetics 139: 1347-1358.

Simcox, A. 1997. Differential requirements for EGF-likeligands in Drosophila wing development. Mech. Dev. 62: 41-50.

Simcox, A.A., G. Grumbling, B. Schnepp, C. Bennington-M athias, E. Hersperger, and A. Shearn. 1996. Molecular, phenotypic, and expression analysis of vein, a gene required for growth of the Drosophila wing disc. Dev. Biol. 177: 475-489.

Smith, J.C., B.M.J. Price, K. Van N immen, and D. Huylebroeck. 1990. Identification of a potent Xenopus mesoderm inducing factor as activin A. Nature 345: 729-731.

Spencer, F.A., F.M. Hoffmann, and W.M. Gelbart. 1982. Decapentaplegic: A gene complex affecting morphogenesis in Drosophila melanogaster. Cell 28: 451-461.

Staehling-Hampton, K. and F.M. Hoffmann. 1994. Ectopic decapentaplegic in the Drosophila midgut alters the expression of five homeotic genes, dpp, and wingless, causing specific morphological defects. Dev. Biol. 164: 502-512.

Sun, B., D.A. Hursh, D. Jackson, and P.A. Beachy. 1995. Ultrabithorax protein is necessary but not sufficient for full activation of decapentaplegic expression in the visceral mesoderm. EMBO J. 14: 520-535.

Szüts, D., M. Freeman, and M. Bienz. 1997. Antagonism between EGFR and Wingless signalling in the larval cuticle of Drosophila. Development 124: 3209-3219.
Thüringer, F. and M. Bienz. 1993. Indirect autoregulation of a homeotic Drosophila gene mediated by extracellular signalling. Proc. Natl. Acad. Sci. 90: 3899-3903.

Thüringer, F., S.M. Cohen, and M. Bienz. 1993. Dissection of an indirect autoregulatory response of a homeotic Drosophila gene. EMBO J. 12: 2419-2430.

Tio, M., C. Ma, and K. M oses. 1994. spitz, a Drosophila homolog of transforming growth factor-al pha, is required in the founding photoreceptor cells of the compound eye facets. Mech. Dev. 48: 13-23.

Tremml, G. 1991. "Interaktionen homeotischer Gene in inneren Keimblättern des Drosophila Embryos." Ph.D. thesis, University of Zürich, Switzerland.

Tremml, G. and M. Bienz. 1989. Homeotic gene expression in the visceral mesoderm of Drosophila embryos. EMBO J. 8: $2677-2685$.

- - 1 1992. Induction of Iabial expression in the Drosophila endoderm: Response el ements for dpp signalling and for autoregulation. Development 116: 447-456.

Wappner, P., L. Gabay, and B.-Z. Shilo. 1997. Interactions between the EGF receptor and DPP pathways establish distinct cell fates in the tracheal placodes. Development 124: 47074716.

White, R.A.H. and M. Wilcox. 1984. Protein products of the bithorax complex in Drosophila. Cell 39: 163-171.

Yarnitzky, T., L. M in, and T. Volk. 1997. The Drosophila neuregulin homolog Vein mediates inductive interactions be tween myotubes and their epidermal attachment cells. Genes \& Dev. 11: 2691-2700.

Yingling, J.M., M.B. Datto, C. Wong, J.P. Frederick, N.T. Liberati, and X.-F. Wang. 1997. Tumor suppressor Smad4 is a transforming growth factor $\beta$-inducible DNA binding protein. Mol. Cell. Biol. 17: 7019-7028.

Yu, X., S. Hoppler, S. Eresh, and M. Bienz. 1996. decapentaplegic, a target gene of the wingless signalling pathway in the Drosophila midgut. Development 122: 849-858.

Zak, N.B., R.J. Wides, E.D. Schejter, E. Raz, and B.-Z. Shilo. 1990. Localization of the DER/flb protein in embryos: Implication on the faint little ball lethal phenotype. Development 109: 865-874.

Zecca, M., K. Basler, and G. Struhl. 1995. Sequential organizing activities of engrailed, hedgehog and decapentaplegic in the Drosophila wing. Development 121: 2265-2278. 


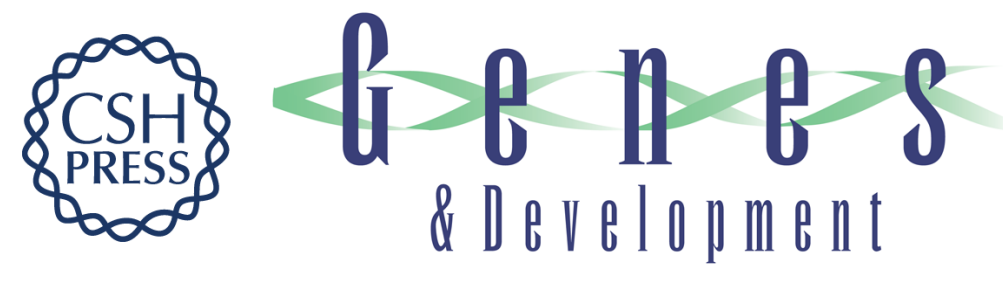

\section{Functional intertwining of Dpp and EGFR signaling during Drosophila endoderm induction}

Dávid Szüts, Salih Eresh and Mariann Bienz

Genes Dev. 1998, 12:

Access the most recent version at doi:10.1101/gad.12.13.2022

References This article cites 87 articles, 41 of which can be accessed free at: http://genesdev.cshlp.org/content/12/13/2022.full.html\#ref-list-1

License

Email Alerting Receive free email alerts when new articles cite this article - sign up in the box at the top Service right corner of the article or click here.

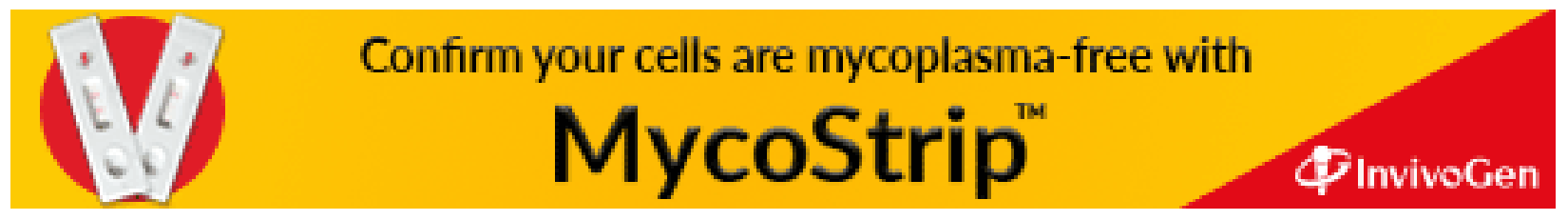

